\title{
Brain regions supporting verbal memory improvement in healthy older subjects
}

\author{
Regiões do cérebro relacionadas à melhora da memória verbal em idosos saudáveis \\ Eliane C. Miotto', Joana B. Balardin', Cary R. Savage², Maria da Graça M. Martin ${ }^{3}$, Marcelo C. Batistuzzo?, \\ Edson Amaro Junior ${ }^{3}$, Ricardo Nitrini ${ }^{1}$
}

\begin{abstract}
Despite growing interest in developing cognitive training interventions to minimize the aging cognitive decline process, no studies have attempted to explore which brain regions support the application of semantic strategies during verbal memory encoding. Our aim was to investigate the behavioral performance and brain correlates of these strategies in elderly individuals using fMRI in healthy older subjects. Method: Subjects were scanned twice on the same day, before and after, directed instructions to apply semantic strategies during the encoding of word lists. Results: Improved memory performance associated to increased semantic strategy application and brain activity in the left inferior and middle and right medial superior prefrontal cortex were found after the directed instructions. There was also reduced activation in areas related to strategy mobilization. Conclusion: Improved memory performance in older subjects after the application of semantic strategies was associated with functional brain reorganization involving regions inside and outside the typical memory network.
\end{abstract}

Keywords: older subjects, semantic strategies, fMRI, episodic memory.

\section{RESUMO}

Apesar do crescente interesse em intervenções de treinamento cognitivo para minimizar o declínio cognitivo do envelhecimento, nenhum estudo explorou quais regiões do cérebro estão relacionadas à aplicação de estratégias semânticas durante a codificação da memória verbal. Nosso objetivo foi investigar o comportamento e correlatos cerebrais associados a essas estratégias usando fMRI em idosos saudáveis. Método: Os sujeitos foram examinados no mesmo dia, antes e depois, de instruções dirigidas para aplicar estratégias semânticas durante a codificação de palavras. Resultados: Melhora da memória relacionada ao uso de estratégias semânticas e aumento da atividade no córtex prefrontal inferior e medial esquerdo e medial superior direito foram encontrados após as instruções. Também houve redução de ativação em áreas de mobilização de estratégias. Conclusão: A melhora da memória em idosos após o uso de estratégias semânticas estava associada à reorganização cerebral funcional envolvendo regiões dentro e fora da rede de áreas cerebrais típicas da memória.

Palavras-chave: idosos, estratégias semânticas, fMRI, memória episódica.

The aging process is associated with progressive decline in cognitive abilities such as memory, attention and executive function ${ }^{1,2,3}$. Therefore, older subjects commonly experience functional and cognitive losses which can affect quality of life, social interaction, and occupational performance. More recently, there has been a great concern with benign cognitive decline and the risk for developing mild cognitive impairment (MCI) or dementia progression. As a consequence, there has been growing interest to explore non-pharmacological clinical interventions, particularly cognitive training, to minimize this process or improve some cognitive skills in older adults ${ }^{4}$.
Episodic memory is the most commonly affected type of memory in aging 5 .. The term "episodic memory" refers to a system responsible for the encoding, storing, and retrieving of personally experienced and highly temporally specific information ${ }^{7}$. Combined evidence from cognitive psychology, neuropsychological and functional neuroimaging studies suggested that efficient episodic memory encoding might engage information processing within specific cortical regions that provides a critical input to medial temporal cortex. Task-related fMRI studies supplied further information regarding the functional neuroanatomy of these cognitive processes. For example, engagement of portions of the dor-

${ }^{1}$ Departamento de Neurologia, Faculdade de Medicina, Universidade de São Paulo, Sao Paulo SP, Brazil;

${ }^{2}$ Center for Health Behavior Neuroscience, Kansas University, Kansas, EUA;

${ }^{3}$ Departamento de Radiologia, Faculdade de Medicina, Universidade de São Paulo, Sao Paulo SP, Brazil.

Correspondence: Departamento de Neurologia, Faculdade de Medicina, Universidade de São Paulo; Av. Dr. Enéas de Carvalho Aguiar, 255; 05403-900 São Paulo SP, Brasil; E-mail: ecmiotto@usp.br

Conflict of interest: There is no conflict of interest to declare.

Received 17 January 2014; Received in final form 16 June 2014; Accepted 07 July 2014. 
solateral prefrontal and the posterior parietal cortices have been reported during working memory tasks ${ }^{8}$, which generally modulates episodic memory performance by promoting efficient encoding through manipulation and monitoring of information to subsequent retrieval ${ }^{9}$. The left ventrolateral prefrontal (VLPFC) cortex has been implicated in the access to the semantic memory system in the temporal pole ${ }^{10}$, which is involved in modulating the depth and organization of material at encoding.

Encoding strategies, used to enhance episodic memory performance are also reduced in aging possibly due to changes in prefrontal structures and their connections with posterior cortical areas such as temporal and parietal cortex which are involved in episodic memory encoding and retrieval $^{11}$. The participation of the prefrontal cortex (PFC) in executive strategic processes, learning and episodic memory is well documented by brain lesion and functional neuroimaging studies ${ }^{12,13}$. One of the encoding strategies known to improve episodic verbal memory is semantic categorization. In typical verbal learning tasks, participants study lists of words and subsequently recall the studied items in the order they come to mind without external cues. But when the studied material is composed by lists of related words, participants have a tendency to recall semantically related items consecutively. Although differing accounts have attributed such effects to associative automatic versus strategic controlled mechanisms operating at encoding, retrieval, or both $^{14,15}$, this effect, named semantic clustering ${ }^{16}$, has been used as an index of strategic episodic learning. The left inferior prefrontal cortex (IPFC) mediates semantic processing of information ${ }^{17,18}$. The dorsolateral prefrontal cortex (DLPFC) supports updating, sequencing, and monitoring operations, all features of working memory, along with attention $^{19}$. The orbitofrontal cortex (OFC) plays an important role in initiating relevant behavior, suppressing irrelevant responses and in the early mobilization of efficient strategies in novel situations ${ }^{20}$. Therefore, the PFC plays an important role at the encoding, organization and strategy creation during memory functioning and this role seems to increase with age ${ }^{5}$.

It has been suggested that older adults are less likely than young adults to use efficient strategies when performing memory tasks although this difference can be reduced by cognitive training ${ }^{21}$. However, direct evidence of the neural mechanisms and brain regions related to memory improvement in healthy older adults are scarce. Yet, brain imaging studies can contribute to our understanding of the aging brain. To date, only few studies have investigated the brain correlates after cognitive training in healthy older subjects. In one such study using positron emission tomography (PET), older adults' brain activity was assessed during encoding before and after the loci training - a mnemonic method to encode words which requires visualization of a series of items in familiar locations ${ }^{22}$. Older adults showed greater activity after training in occipitoparietal cortex related to improved performance. Using the same loci method in an 8-week cognitive training, older participants showed increased cortical thickness, as measured by magnetic resonance imaging (MRI), in the right orbitofrontal gyrus, insula, and fusiform gyrus ${ }^{23}$. In a functional MRI (fMRI) study, 16 older adults received two training sessions, in different days, associating pleasantness, personal relevance, and sentence creation to encode words ${ }^{21}$. There was increased activity during encoding in the medial superior frontal gyrus, right precentral gyrus, and left caudate after training. Other recent studies have examined the neural correlates of cognitive training in both healthy aging and MCI subjects. In one of them, increased activation was found after a 6-week cognitive training in the MCI group encompassing a large network including the frontal, temporal and parietal regions whereas healthy controls showed mostly areas of decreased activation following training ${ }^{24}$. In another recent brain imaging study ${ }^{25}$, MCI and healthy controls were scanned as they encoded and retrieved object location associations - a visuospatial memory task. Although the fMRI analyses were restricted to the hippocampus, following training, the MCI group showed increased left and right hippocampi activity at encoding and retrieval, respectively and the healthy older controls showed mainly decreased activation after training in the right hippocampus.

Despite these previous results, to our knowledge, there are no studies exploring which brain regions, particularly involving the frontal networks, are engaged during the application of semantic organizational strategies in healthy older subjects and what are their impact on memory performance. Therefore, the aims of the current study were to investigate ${ }^{1}$ the brain correlates of semantic strategy application during verbal memory encoding in older adults using fMRI, and ${ }^{2}$ the effects of this strategy on memory performance.

\section{METHOD}

\section{Participants}

Seventeen community-dwelling healthy older adults were enrolled in the study. Participants were initially assessed by screening and standardized cognitive tests of memory, attention, executive, language and intellectual functioning, mood questionnaire, and structural magnetic resonance imaging. They were all right-handed and native BrazilianPortuguese speakers. Table 1 presents the demographic and behavioural characteristics of the participants. Average performance on all cognitive measures was within the normal range adjusted for age and education. Potencial participants were excluded if they reported history of neurological 
Table 1. Participants' clinical and neuropsychological characteristics. Results are expressed as meantstandard deviation.

\begin{tabular}{lc}
\hline Variable & $\begin{array}{c}\text { Healthy } \\
\text { older adults }\end{array}$ \\
\hline Age & $68.12(1.19)$ \\
Years of formal education & $10.94(1.30)$ \\
Gender & $10 F / 7 \mathrm{M}$ \\
MMSE & $28.31(0.35)$ \\
GDS & $1.24(0.35)$ \\
IADLs3 & $0.31(0.19)$ \\
Estimated Full Scale IQ & $104.56(2.28)$ \\
Letter fluency - FAS* & $38.77(2.42)$ \\
Animal fluency* & $23.55(2.21)$ \\
Stroop task - color card (time in sec.) & $15.01(0.69)$ \\
Stroop task - Word card (time in sec.) & $21.57(1.72)$ \\
Stroop task - color/word card (time in sec.) & $30.96(2.03)$ \\
Trail Making - part A (time in sec.) & $47.91(7.41)$ \\
Trail Making - part B (time in sec.) & $128.82(19.80)$ \\
Logical Memory immediate recall & $25.80(1.42)$ \\
Logical Memory delayed recall & $23.93(1.26)$ \\
RAVLT total & $45.81(2.88)$ \\
RAVLT immediate recall & $9.44(0.65)$ \\
RAVLT delayed recall & $8.81(0.65)$ \\
Rey-Osterrieth Complex Figure copy** & $31.57(1.09)$ \\
Rey-Osterrieth Complex Figure delayed recall** & $15.00(1.72)$ \\
Digit span forward & $7.23(0.48)$ \\
Digit span backward & $5.15(0.43)$ \\
\hline MMSE: Mini-Mental State Examination; GDS: Geratic Depression
\end{tabular}

MMSE: Mini-Mental State Examination; GDS: Geriatric Depression Scale; Instrumental Activities of Daily Living Scale (Pfeffer); Estimated Full Scale IQ based on the Weschler Adult Intelligence Scale III; RAVLT: Rey Auditory Verbal Learning Test, sum of words recalled across the 5 consecutive trials; Letters selected to prompt word generation; *Total number of

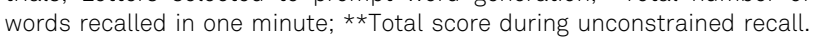

or psychiatric disease, cognitive complaints or deficits identified by the neuropsychological tests based on their norms. Exclusion criteria included the use of any psychotropic medication (except antidepressants in stable doses) within the previous 6 months, and previous or current use of illegal psychoactive drugs. In addition, the exclusion criteria included vascular lesions, hippocampus or cerebral atrophy identified by the structural MRI, uncontrolled hypertension, unstable medical conditions (i.e., diabetes, hypothyroidism, vitamin B12 or folate deficiency), and presence of any metal in their body other than dental fillings. The study was approved by the local ethics committee (CAPPesq 06/03336).

Study design and fMRI experimental paradigm participants were scanned using a block design during the encoding of word lists: 16 unrelated words (UR) and 16 semantically related words (SR) in terms of semantic categories, alternated with a fixation baseline (+) (Figure 1). The current paradigm was based on the California Verbal Learning Test (CVLT) ${ }^{26}$ and validated in healthy adults and patients with brain lesions using fMRI ${ }^{19,27}$. For the UR condition, the words presented were not semantically related making it difficult to apply the semantic organizational strategies. For the SR condition, the words were semantically related into four categories and were presented in a randomized mixed order (Figure 1). The word lists were generated from 32 categories of words with four words in each category. The paradigm was tested extensively in pilot studies in order to develop word lists that prompted significant

\begin{tabular}{|c|c|c|c|c|c|c|c|c|c|c|c|c|}
\hline Fix1 & & Fix2 & & Fix3 & & Fix4 & & Fix5 & & Fix6 & & Fix7 \\
\hline+ & $\mathrm{SR}$ & + & UR & + & SR & + & UR & + & UR & + & SR & + \\
\hline $12 s$ & $33 s$ & $12 \mathrm{~s}$ & $33 s$ & $12 \mathrm{~s}$ & $33 s$ & $12 s$ & $33 s$ & $12 s$ & $33 s$ & $12 \mathrm{~s}$ & $22 s$ & $12 s$ \\
\hline \multicolumn{3}{|c|}{ Categories } & & \multicolumn{4}{|l|}{$\mathrm{SR}$} & \multicolumn{5}{|c|}{ UR } \\
\hline \multirow{16}{*}{\multicolumn{3}{|c|}{$\begin{array}{l}\text { Body parts } \\
\text { (Land animals) } \\
\text { Cleaning supplies } \\
\text { (Water animals) }\end{array}$}} & & \multicolumn{5}{|l|}{ Eye } & \multicolumn{4}{|c|}{ Lemon } \\
\hline & & & & \multicolumn{5}{|c|}{ Penguin } & \multicolumn{4}{|c|}{ Comb } \\
\hline & & & & \multicolumn{5}{|c|}{ Dustpan } & \multicolumn{4}{|c|}{ Ship } \\
\hline & & & & \multicolumn{5}{|c|}{ Oyster } & \multicolumn{4}{|c|}{ Cake } \\
\hline & & & & \multicolumn{5}{|c|}{ Detergent } & \multicolumn{4}{|c|}{ Fork } \\
\hline & & & & \multicolumn{5}{|c|}{ Squid } & \multicolumn{4}{|c|}{ Piano } \\
\hline & & & & \multicolumn{5}{|c|}{ Beaver } & \multicolumn{4}{|c|}{ Shirt } \\
\hline & & & & \multicolumn{5}{|c|}{ Finger } & \multicolumn{4}{|c|}{ Salt } \\
\hline & & & & \multicolumn{5}{|l|}{ Cod } & \multicolumn{4}{|c|}{ Doll } \\
\hline & & & & \multicolumn{5}{|c|}{ Sponge } & \multicolumn{4}{|c|}{ Blocks } \\
\hline & & & & \multicolumn{5}{|l|}{ Deer } & \multicolumn{4}{|c|}{ Table } \\
\hline & & & & \multicolumn{5}{|c|}{ Ankle } & \multicolumn{4}{|c|}{ Paper } \\
\hline & & & & \multicolumn{5}{|c|}{ Bucket } & \multicolumn{4}{|c|}{ Water } \\
\hline & & & & \multicolumn{5}{|c|}{ Salmon } & \multicolumn{4}{|c|}{ Window } \\
\hline & & & & \multicolumn{5}{|c|}{ Nose } & & aint & & \\
\hline & & & & Wolf & & & & & & able & & \\
\hline
\end{tabular}

SR: semantically related words; UR: unrelated words.

Figure 1. Experimental paradigm including seven fixation periods (Fix) interleaved with 3 repetitions of each list (SR, UR) with 16 words. Example of the list words (SR and UR). 
differences in semantic clustering. In addition, the inclusion of categories that shared "fuzzy" semantic relationships (e.g., land animals and water animals) made the task more challenging and minimized ceiling effects for clustering. Scanning was carried out twice: before and after a brief period of directed instructions and practice (30-min) to apply the semantic organizational strategies. In the first fMRI session (before the directed instructions), subjects were instructed to look at the words presented onto the screen and try to remember as many words as they could, without a specific instruction to apply any strategy. After the first fMRI session, they were taken to a different room and submitted to a training consisted of teaching them to apply semantic organizational strategies to a set of different word lists. Subjects were taught to organize a set of different word lists into categories and then to memorize and retrieve the words according to each category. The instructions for the orientation training were as follows: "Notice that the words appearing on the screen can be grouped into four different categories. By grouping the words together into these related categories, you will remember more words. For example, in this list you can group the words into body parts, land animals, cleaning supplies and water animals. The words can be grouped into four categories. Be sure and group the words together into categories this time as you learn them". Immediately after and on the same day, they were taken to the scanner and instructed to look at novel words presented onto the screen and apply the semantic organizational strategy. As in the first fMRI session, following the scan, participants were prompted to recall as many words as they could remember in any order and this measure was used as free recall. It should be noted that for all conditions including those in the first and second fMRI sessions we used novel words in order to avoid practice effects. Semantic clustering index scores were defined as consecutive recall of two words from the same category. They reflected the proportion of clustered responses out of the total possible clusters for that condition defined as [clusters/ (words recalled-categories recalled)]. Differences between scanning sessions in free recall scores and semantic clustering index were examined using a paired-sample t-test. All stimuli were visually presented on a screen and synchronized with the scanner via an optic relay triggered by the radiofrequency pulse. The presentation order of the words in each list was randomized and conditions (SR, UR) were counterbalanced within scans and across participants.

Image acquisition and fMRI analysis scans were performed on a Philips 3 tesla Achieva MRI scanner (8ch head coil). The fMRI acquisition was based on $\mathrm{T} 2 *$-weighted echo planar (EPI) images for the whole brain with the following parameters: TR=3,000 ms, TE $=30 \mathrm{~ms}, 41$ slices, $3 \mathrm{~mm}$ isotropic voxel size, FOV=240 mm and matrix $80 \times 78$, with 94 volumes, discarding the initial four scans in each run to allow steady-state magnetization to be approached. There were two runs, each run with 32 words and a total number of words equal to 64 (all novel words). Each run lasted $282 \mathrm{~s}$ (4min42s), in which were collected 94 volumes covering all the brain.

FSL package (version 4.1, FMRIB Analysis Group, www. fmrib.ox.ac.uk/fsl) was used to analyze the fMRI data. Preprocessing included movement correction (MCFLIRT), spatial smoothing using a $5 \mathrm{~mm}$ Gaussian kernel, and spatial normalization to MNI-152 template with a 12 DoF affine registration (FLIRT). The general linear model (GLM) with residual autocorrelation correction (FILM) was applied to obtain statistical activation maps. The design matrix was composed of regressors according to the experimental conditions (SR and UR). For each condition, the haemodynamic response function in GLM was determined considering the convolution of experimental designs with a gamma function $(\mathrm{SD}=3 \mathrm{~s}$, mean lag=6 s). The group activation maps and session comparisons were obtained using the mixed-effects model. Contrast images for each encoding condition (SR $>$ fixation, UR $>$ fixation) in the first and second fMRI sessions were created for each subject. In a preliminary analysis, the contrasts $\mathrm{SR}>$ fixation and UR $>$ fixation were entered into a second-level analysis [FMRIB's Local Analysis of Mixed Effects-FLAME stage $1^{28}$ to test if there were a main effect of list (SR, UR) or a list session (spontaneous, directed) interaction, in order to identify regions whose activation increased or decreased between sessions, more for the SR contrast than the NR contrast. There were no brain regions that showed a significant interaction or a main effect of list. Therefore, because the focus of the present study was on identifying session-related activation that are associated to strategic behaviour, the remaining analyses focused only in the contrast SR $>$ fixation, giving the sensitivity of the SR word list in prompting measurable semantic clustering scores. These contrast images were then used for betweensessions comparisons (paired-sample t-test) in order to obtain the brain activation pattern change over time. All the statistical images were thresholded by using Gaussian random field-based cluster inference with a threshold of $\mathrm{Z}>2.3$ at the voxel level and a corrected cluster significance threshold of $\mathrm{P}<0.05$.

\section{RESULTS}

\section{Behavioural results}

The results shown on Table 2 indicate that subjects used significantly more verbal learning strategies after the directed instructions to apply the semantic strategies demonstrated by higher semantic clustering index scores for the SR condition $(\mathrm{t}=-4.006, \mathrm{p}=0.001)$. In addition, there was a significant correlation between semantic clustering index scores after the 
Table 2. Performance on free recall, semantic clustering index and recognition for each condition during the first and second fMRI sessions. Results are expressed as mean \pm standard deviation.

\begin{tabular}{lcc}
\hline & $1^{\text {st }}$ session & $2^{\text {nd }}$ session \\
\hline Free recall & & \\
SR* & $7.53(0.575)$ & $9.41(0.810)$ \\
UR* & $3.59(0.613)$ & $3.29(0.391)$ \\
Semantic clustering index & $0.242(0.044)$ & $0.452(0.038)$ \\
Number of clusters $(\max 12)$ & $3.23(0.465)$ & $4.58(0.675)$ \\
Recognition & & \\
$\quad$ Hit rate SR & $0.92(0.022)$ & $0.82(0.036)$ \\
$\quad$ Hit rate UR & $0.83(0.036)$ & $0.87(0.026)$ \\
$\quad$ Corrected rejection rate & $0.92(0.029)$ & $0.89(0.023)$ \\
\hline
\end{tabular}

*Total number of words recalled; SR: semantic related list; UR: semantic unrelated list.

directed instructions and the total number of words recalled only after training for the SR condition $(r=0.652, p=0.002)$. In terms of word free recall, there was a significant change only for the SR condition $(\mathrm{t}=-3.665, \mathrm{p}=0.002)$ with more words recalled after the directed instructions.

Neuroimaging results are presented in Figure 2. Participants exhibited increased activation in the $2^{\text {nd }}$ fMRI session compared to the $1^{\text {st }}$ fMRI for the contrast SR $>$ baseline in clusters encompassing left inferior frontal gyrus, middle frontal gyrus and precentral gyrus, left intraparietal sulcus, angular gyrus, left cerebellum and right medial superior frontal gyrus and anterior cingulate. When comparing the $1^{\text {st }}>2^{\text {nd }}$ fMRI, decreased activation was observed in the bilateral posterior cingulate and left precuneus, right medial superior frontal gyrus and anterior cingulate, right inferior parietal lobule and middle and superior temporal cortex, right dorsal superior frontal gyrus, and bilateral orbitofrontal cortex (Figures 2 and 3; Table 3).

Results for the UR>baseline showed increased activation in the $2^{\text {nd }}$ fMRI session in left superior frontal gyrus and precentral gyrus, bilateral precuneus and inferior parietal lobule, bilateral medial superior frontal gyrus and right anterior cingulate. Decreased activation was observed in the right superior frontal gyrus, medial orbital gyrus and anterior cingulate, bilateral lingual gyrus, left cuneus and posterior cingulate, and bilateral precuneus (Figures 2 and 3; Table 3).

\section{DISCUSSION}

The present study investigated the neural correlates of semantic strategy application during memory encoding in older adults using fMRI. The main behavioral results showed a significant increase in semantic strategy application after the directed instructions and practice to apply these strategies, suggesting that older adults are able to learn and implement efficient strategies. In addition, participants recalled more words after the directed instructions for the SR condition and there was a significant correlation between semantic strategy scores and words recalled for the SR condition, a condition where this strategy can be used. These findings are similar to the ones found in healthy adult subjects ${ }^{19}$ and other studies using different cognitive training in elderly subjects ${ }^{21,22}$ and show that after a brief period of practice to apply efficient
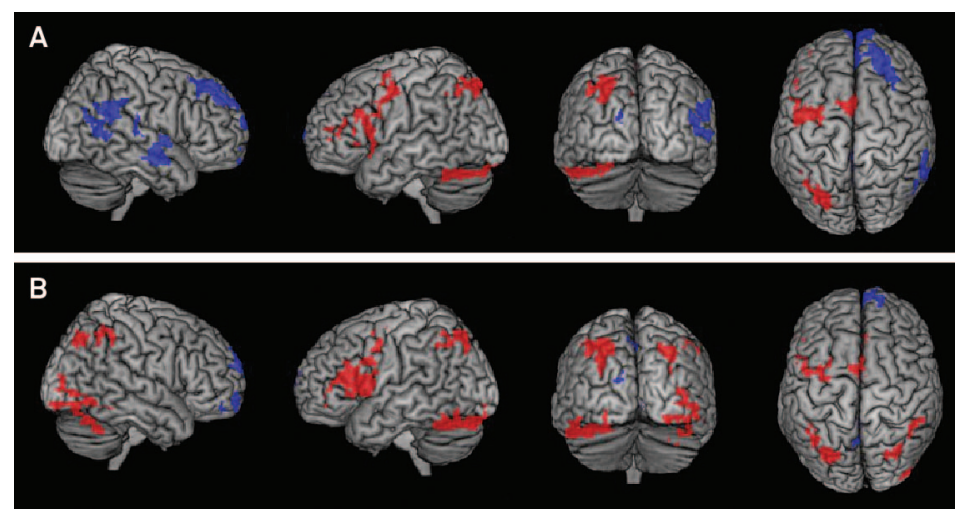

Figure 2. Brain regions with significant differences between the first and second fMRI sessions. Regions in which increased fMRI BOLD signal was observed in the second scan compared with the first one are depicted in red and regions with decreased activation are depicted in blue. (A) Contrast SR>baseline in red: left inferior frontal gyrus, middle frontal gyrus and precentral gyrus (cluster 1), left intraparietal sulcus and angular gyrus (cluster 2), right medial superior frontal gyrus and anterior cingulate (cluster 3), left cerebellum (cluster 4). In blue: bilateral posterior cingulate and left precuneus (cluster 1), right superior frontal gyrus and anterior cingulate (cluster 2), right inferior parietal lobule and middle and superior temporal cortex (cluster 3), right dorsal superior frontal gyrus (cluster 4), and bilateral orbitalfrontal cortex (cluster 5). (B) Contrast UR>baseline in red: left superior frontal gyrus and precentral gyrus (cluster 1), bilateral precuneus and inferior parietal lobule (clusters 2 and 3), bilateral medial portion of superior frontal gyrus and right anterior cingulate (cluster 4). In blue: right superior frontal gyrus, medial orbital gyrus and anterior cingulate (cluster 1), bilateral lingual gyrus and left cuneus and posterior cingulate (cluster 2), and bilateral precuneus (cluster 3). 

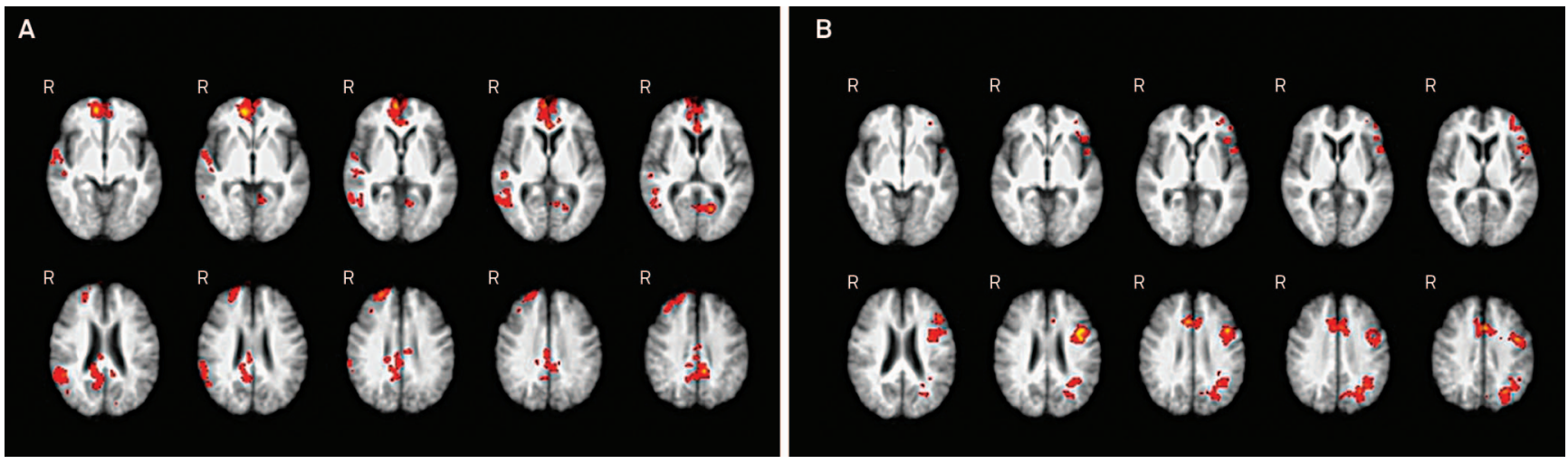

Figure 3. Brain regions with significant differences for the contrasts $A 1^{\text {st }}>2^{\text {nd }} \mathrm{fMRI}$ and $\mathrm{B} 1^{\text {st }}<2^{\text {nd }}$ fMRI in the contrast $\mathrm{SR}>$ fixation.

Table 3. Significant clusters highlighted when comparing the BOLD response between the spontaneous and directed use of learning strategies during word list encoding compared to fixation baseline.

\begin{tabular}{|c|c|c|c|c|c|c|c|}
\hline \multirow[t]{2}{*}{ Contrast } & \multirow[t]{2}{*}{ Side } & \multicolumn{3}{|c|}{ MNI coordinates } & \multirow[t]{2}{*}{$Z$ value } & \multirow[t]{2}{*}{ Cluster size } & \multirow[t]{2}{*}{ Cluster $p$-value } \\
\hline & & $x$ & $\mathrm{y}$ & z & & & \\
\hline \multicolumn{8}{|l|}{$2^{\text {nd }} \mathrm{fMRI}>1^{\text {st }} \mathrm{fMRI}$} \\
\hline \multicolumn{8}{|l|}{ Region } \\
\hline VLPFC & & & & & & 2131 & $<0.001$ \\
\hline Inferior frontal gyrus (BA 44) & $\mathrm{R}$ & -42 & 10 & 32 & 4.6 & & \\
\hline Inferior frontal gyrus (BA 45) & $\mathrm{R}$ & -46 & 24 & 24 & 4.25 & & \\
\hline midDLPFC & & & & & & & $<0.001$ \\
\hline Middle frontal gyrus (BA 46) & $\mathrm{R}$ & -36 & 0 & 54 & 4.04 & & \\
\hline Precentral gyrus (BA 6) & $\mathrm{R}$ & -42 & 0 & 32 & 4.17 & & \\
\hline Posterior Parietal Cortex & & & & & & 1021 & $<0.001$ \\
\hline Intraparietal sulcus (BA 7) & $\mathrm{R}$ & -24 & -70 & 48 & 4.27 & & \\
\hline Angular gyrus (BA 40) & $\mathrm{R}$ & -34 & -58 & 40 & 3.58 & & \\
\hline Pre-SMA & & & & & & 977 & $<0.001$ \\
\hline Superior frontal gyrus (BA 6) & $\mathrm{R}$ & -6 & 2 & 58 & 4.66 & & \\
\hline Cingulate gyrus (BA 32) & $\mathrm{R}$ & 6 & 20 & 36 & 3.86 & & \\
\hline Cerebelum & $\mathrm{R}$ & -40 & -76 & -26 & 4.27 & 545 & 0.003 \\
\hline \multicolumn{8}{|l|}{$1^{\text {st }} \mathrm{fMRI}>2^{\text {nd }} \mathrm{fMRI}$} \\
\hline \multicolumn{8}{|l|}{ Region } \\
\hline Precuneus/Posterior cingulate & & & & & & 1894 & $<0.001$ \\
\hline Posterior cingulate (BA 30) & $\mathrm{R}$ & -16 & -66 & 12 & 3.97 & & \\
\hline Precuneus (BA 7) & $\mathrm{R}$ & 0 & -54 & 46 & 3.83 & & \\
\hline mPFC & & & & & & 1710 & $<0.001$ \\
\hline Superior frontal gyrus (BA 10) & $\mathrm{R}$ & 12 & 54 & -4 & 4.37 & & \\
\hline Anterior cingulate gyrus (BA 24) & $\mathrm{R}$ & 0 & 36 & 8 & 3.77 & & \\
\hline Inferior temporal & & & & & & 896 & $<0.001$ \\
\hline Inferior parietal lobule (BA 39) & $\mathrm{R}$ & 50 & -48 & 24 & 4.16 & & \\
\hline Supramarginal gyrus (BA 40) & $\mathrm{R}$ & 60 & -50 & 30 & 3.69 & & \\
\hline Middle temporal gyrus (BA 21) & $\mathrm{R}$ & 52 & -56 & 6 & 3.34 & & \\
\hline Superior temporal gyrus (BA 22) & $\mathrm{R}$ & 52 & -44 & 10 & 3.26 & & \\
\hline Superior temporal & & & & & & 619 & 0.001 \\
\hline Middle temporal gyrus (BA 21) & $\mathrm{R}$ & 64 & -6 & -16 & 3.74 & & \\
\hline Superior temporal gyrus (BA 22) & $\mathrm{R}$ & 56 & -26 & 8 & 3.72 & & \\
\hline Superior frontal & & & & & & 528 & 0.004 \\
\hline Superior frontal gyrus (BA 9) & $\mathrm{R}$ & 24 & 40 & 46 & 3.77 & & \\
\hline Middle frontal gyrus (BA 46) & $\mathrm{R}$ & 26 & 46 & 30 & 3.35 & & \\
\hline OFC & & & & & & 421 & 0.017 \\
\hline Middle orbital gyrus (BA11) & $\mathrm{R}$ & 18 & 16 & -22 & 3.5 & & \\
\hline Rectus gyrus (BA 11) & $\mathrm{R}$ & -6 & 6 & -22 & 3.1 & & \\
\hline
\end{tabular}

FMRI: functional magnetic resonance imaging; VLPFC: left ventrolateral prefrontal; DLPPC: dorsolateral prefrontal cortex; BA: Brodmann area; SMA: supplementary motor area; PFC: prefrontal cortez; OFC: orbitofrontal cortex. 
strategies older subjects can substantially improve their memory performance.

Brain imaging results for the SR condition, in which semantic processing and active organization in working memory were most demanded in relation to the UR condition, indicated increased activation in the second fMRI session compared to the first in the left inferior and middle frontal gyrus, precentral gyrus, intraparietal sulcus, angular gyrus, cerebellum and in the right medial superior frontal gyrus and anterior cingulate. These findings are in line with studies of healthy older subjects employing longer periods of cognitive training showing increased activity after training in bilateral prefrontal regions including the inferior and medial frontal gyrus ${ }^{21,23,25}$. Previous results suggested that the left middle and inferior frontal gyrus and cerebellum support young adults' verbal encoding strategies ${ }^{19,27}$. In addition, there was greater activity in the right medial superior frontal gyrus and anterior cingulate. Semantic categorization strategy training has been associated to increased brain activity in the right middle and inferior frontal gyri in healthy adults $^{19}$. Taken together these findings suggest that semantic organizational strategy application is associated with bilateral PFC increased activity regardless of age.

Decreased activation was noted in bilateral OFC, right medial and dorsal superior frontal gyrus, inferior parietal lobule, anterior and posterior cingulate, left precuneus, middle and superior temporal cortex. Here again, these results are similar to studies with healthy young adults showing decreased activation in the OFC after training or instruction to apply semantic organizational strategies ${ }^{19,27,28}$. Previous investigations indicated that the OFC mediates memory processes by mobilizing effective strategies in novel situations $^{18,29}$. It is possible that, after the instructions to apply the semantic strategies, this ability became consolidated resulting in OFC decreased activation. Areas of reduced activity were also found in the right DLPFC, medial frontal cortex and anterior cingulate after the instructions to apply the semantic strategies. These findings were possibly related to the reduced attentional demands after these instructions. These cerebral regions have previously been associated with attention and monitoring processes and patients with lesions in these areas are usually impaired in tasks requiring sustained attention and working memory ${ }^{12}$.

Participants supressed the responses of $\mathrm{mPFC}$, precuneous and bilateral posterior cinulate encoding after the explicit orientation to apply the verbal learning strategy compared to the first fMRI session. In the context of task induced deactivation studies, these regions, along inferior parietal lobule, have been consistently reported as nodes of the default mode network (DMN) of the brain ${ }^{30}$. Although the identification of a possible DMN was originally identified as a phenomenon whereby a set of regions exhibited greater blood flow or BOLD signal during baseline resting or fixation conditions than during task performance, subsequent studies demonstrated similar patterns of results when tasks with different cognitive demands were contrasted $^{31}$. Recent studies have shown, for example, that $\mathrm{mPFC}$ activity is greater during performance of a relatively easy 0 -back task than a 2-back working memory task ${ }^{32}$, and that suppressing activity in DMN regions during working memory encoding, was predictive of subsequent better performance ${ }^{33}$. In our study, when an increased working memory demand was imposed by the direct application of the encoding strategy, healthy older adults exhibited a suppression of these regions (i.e. less activation). The exact nature of the DMN suppression, however, is not fully understood. The most common theoretical interpretation has been that "task-induced deactivations" reflect the occurrence of spontaneous internally directed thought processes such as autobiographical memory retrieval and internally oriented mindwandering during unconstrained baseline conditions ${ }^{34}$.

For the UR condition, where application of the semantic strategy proved to be difficult because the words were not related, increased activation after the directed instructions was found in the left superior frontal and gyrus, bilateral precuneus and inferior parietal lobule, bilateral medial portion of superior frontal gyrus and right anterior cingulate. It is possible that these activations were associated with the attempt of the subjects to apply the semantic strategies, even though this would prove to be difficult given that the words were unrelated. It should be noted that participants were not instructed as to which order each condition was presented.

The results of our study should be interpreted in the context of some potential limitations. First, we did not control for repetition effects in the MRI. However, in a previous work of our group ${ }^{35}$ we adopted an approach used by Belleville and cols. $^{24}$ to evaluate the fMRI task repetition effect on brain activation using the same word-list encoding paradigm. In this study ${ }^{35}$ the activations related to the first and second runs acquired in the first fMRI session were compared, so each subject was used as its own control. The results of this analysis demonstrated no significant brain activation changes between the runs, suggesting that repeating the task without the instructions and practice to apply the strategies did not produce measurable repetition or practice effects on brain activation. Another limitation is the lack of a placebo-control condition, which is a challenge in behavioral intervention studies.

In conclusion, the present results demonstrated the engagement of bilateral $\mathrm{PFC}$ and posterior areas during semantic strategy application in a verbal encoding paradigm indicating the recruitment of new, in addition to the typical memory encoding network, consistent with functional reorganization also found in healthy adult subjects ${ }^{19,21,22}$. These findings suggest that a brief period of instructions and prac- 
tice to apply semantic strategies are sufficient to alter older adults' brain activity and behavioural performance. Future longitudinal studies are needed to determine whether this effect persists or changes across time and if it can also be observed in patients with mild cognitive impairment and other cerebral degenerative diseases.

\section{References}

1. Fillit HM, Butler RN, O’Connell AW, et al. Achieving and maintaining cognitive vitality with aging. Mayo Clin Proc 2002;77:681-696.

2. Verhaeghen P, Geraerts N, Marcoen A. Memory complaints, coping, and well-being in old age: a systemic approach. Gerontologist 2000;40:540-548.

3. Bischkopf J, Busse A, Angermeyer MC. Mild cognitive impairment - a review of prevalence, incidence and outcome according to current approaches. Acta Psychiatr Scand 2002;106:403-414.

4. Gross AL, Parisi JM, Spira AP, et al. Memory training interventions for older adults: a meta-analysis. Aging Ment Health 2012;16:722-734.

5. Dennis NA, Hayes SM, Prince SE, Madden DJ, Huettel SA, Cabeza R. Effects of aging on the neural correlates of successful item and source memory encoding. J Exp Psychol Learn Mem Cogn 2008;34:791-808.

6. Fairchild JK, Scogin FR. Training to Enhance Adult Memory (TEAM): an investigation of the effectiveness of a memory training program with older adults. Aging Ment Health 2010;14:364-373.

7. Tulving E. Elements of episodic memory. Oxford [Oxfordshire]: New York: Clarendon Press; Oxford University Press; 1983:351.

8. Champod AS, Petrides M. Dissociation within the frontoparietal network in verbal working memory: a parametric functional magnetic resonance imaging study. J Neurosci 2010;30:3849-3856.

9. Baddeley A. Working memory: theories, models, and controversies. Annu Rev Psychol 2012;63:1-29.

10. Paller KA, Wagner AD. Observing the transformation of experience into memory. Trends Cogn Sci (Regul Ed) 2002;6:93-102.

11. Craik FIM. Levels of processing: past, present. and future? Memory 2002;10:305-318.

12. Stuss DT. Functions of the frontal lobes: relation to executive functions. J Int Neuropsychol Soc 2011;17:759-765.

13. Shallice T, Fletcher P, Frith CD, Grasby P, Frackowiak RS, Dolan RJ. Brain regions associated with acquisition and retrieval of verbal episodic memory. Nature 1994;368:633-635.

14. Gershberg FB, Shimamura AP. Impaired use of organizational strategies in free recall following frontal lobe damage. Neuropsychologia 1995;33:1305-1333.

15. Alexander MP, Stuss D, Gillingham S. Impaired list learning is not a general property of frontal lesions. J Cogn Neurosci 2009;21:1422-1434.

16. Bousfield WA. The occurrence of clustering in the recall of randomly arranged associates. J Gen Psychol 1953;49:229-240.

17. Demb JB, Desmond JE, Wagner AD, Vaidya CJ, Glover GH, Gabrieli JD. Semantic encoding and retrieval in the left inferior prefrontal cortex: a functional MRI study of task difficulty and process specificity. $J$ Neurosci 1995;15:5870-5878.

18. Savage CR, Deckersbach T, Heckers S, et al. Prefrontal regions supporting spontaneous and directed application of verbal learning strategies: evidence from PET. Brain 2001;124:219-231.

19. Miotto EC, Savage CR, Evans JJ, et al. Bilateral activation of the prefrontal cortex after strategic semantic cognitive training. Hum Brain Mapp 2006;27:288-295.
20. Bechara A, Tranel D, Damasio H, Damasio AR. Failure to respond autonomically to anticipated future outcomes following damage to prefrontal cortex. Cereb Cortex 1996;6:215-225.

21. Kirchhoff BA, Anderson BA, Barch DM, Jacoby LL. Cognitive and neural effects of semantic encoding strategy training in older adults. Cereb Cortex 2012;22:788-799.

22. Nyberg L, Sandblom J, Jones S, et al. Neural correlates of trainingrelated memory improvement in adulthood and aging. Proc Natl Acad Sci USA 2003;100:13728-13733.

23. Engvig A, Fjell AM, Westlye LT, et al. Effects of memory training on cortical thickness in the elderly. Neuroimage 2010;52:1667-176.

24. Belleville S, Clément F, Mellah S, Gilbert B, Fontaine F, Gauthier S. Training-related brain plasticity in subjects at risk of developing Alzheimer's disease. Brain 2011;134:1623-1634.

25. Hampstead BM, Stringer AY, Stilla RF, Giddens M, Sathian K. Mnemonic strategy training partially restores hippocampal activity in patients with mild cognitive impairment. Hippocampus 2012:22:1652-1658.

26. Donders J. Performance discrepancies on the California Verbal Learning Test - Second Edition (CVLT-II) in the standardization sample. Psychol Assess 2006;18:458-463.

27. Miotto EC, Savage CR, Evans JJ, et al. Semantic strategy training increases memory performance and brain activity in patients with prefrontal cortex lesions. Clin Neurol Neurosurg 2013;115:309-316.

28. Woolrich MW, Behrens TEJ, Beckmann CF, Jenkinson M, Smith SM. Multilevel linear modelling for FMRI group analysis using Bayesian inference. Neuroimage 2004;21:1732-1747.

29. Nobre AC, Coull JT, Frith CD, Mesulam MM. Orbitofrontal cortex is activated during breaches of expectation in tasks of visual attention. Nat Neurosci 1999;2:11-12.

30. Toro R, Fox PT, Paus T. Functional coactivation map of the human brain. Cereb Cortex 2008;18:2553-2559.

31. Raichle ME, MacLeod AM, Snyder AZ, Powers WJ, Gusnard DA, Shulman GL. A default mode of brain function. Proc Natl Acad Sci USA 2001;98:676-682.

32. Leech R, Kamourieh S, Beckmann CF, Sharp DJ. Fractionating the default mode network: distinct contributions of the ventral and dorsal posterior cingulate cortex to cognitive control. J Neurosci 2011;31:3217-3224.

33. Anticevic A, Repovs G, Barch DM. Resisting emotional interference: brain regions facilitating working memory performance during negative distraction. Cogn Affect Behav Neurosci 2010;10:159-173.

34. Buckner RL, Andrews-Hanna JR, Schacter DL. The brain's default network: anatomy, function, and relevance to disease. Ann N Y Acad Sci 2008;1124:1-38.

35. Miotto EC, Balardin JB, Vieira G, Sato JR, Martin MdGM, et al. Right inferior frontal gyrus activation is associated with memory improvement in patients with left frontal low-grade glioma resection. Plos One 2014;9(8):e105987. 